Article

\title{
Determination of Ochratoxin A in Wheat and Maize by Solid Bar Microextraction with Liquid Chromatography and Fluorescence Detection
}

\author{
Nabil Al-Hadithi ${ }^{1, *}$, Philip Kössler ${ }^{2,3}$ and Petr Karlovsky ${ }^{2}$
}

1 Faculty of Pharmaceutical Sciences, The Hashemite University, Zarqa 13115, Jordan

2 Molecular Phytopathology and Mycotoxin Research, Georg-August-University Göttingen, Grisebachstrasse 6, Göttingen 37077, Germany; E-Mails: philip.koessler@uni-kassel.de (P.K.); pkarlov@gwdg.de (P.K.)

3 Department of Soil Biology and Plant Nutrition, University of Kassel, Nordbahnhofstrasse 1a, Witzenhausen 37213, Germany

* Author to whom correspondence should be addressed; E-Mail: nnahmad@googlemail.com; Tel.: +962-53903333 (ext. 5402).

Academic Editor: Aldo Laganà

Received: 29 March 2015 / Accepted: 31 July 2015 / Published: 5 August 2015

\begin{abstract}
Solid bar microextraction (SBME), followed by liquid chromatography with fluorescence detection (HPLC-FLD), for the quantification of ochratoxin A in wheat and maize was developed. Ground wheat and maize grains were extracted with acetonitrile-water-acetic acid (79:20:1, $v / v / v)$, followed by defatting with cyclohexane, and subjected to SBME-LC-FLD analysis. SBME devices were constructed by packing $2 \mathrm{mg}$ sorbent (C18) into porous polypropylene micro-tubes $(2.5 \mathrm{~cm}$ length, $600 \mu \mathrm{m}$ i.d., and $0.2 \mu \mathrm{m}$ pore size). SBME devices were conditioned with methanol and placed into $5 \mathrm{~mL}$ stirred sample solutions for $70 \mathrm{~min}$. After extraction, OTA was desorbed into $200 \mu \mathrm{L}$ of methanol for $15 \mathrm{~min}$, the solution was removed in vacuum, the residue was dissolved in $50 \mu \mathrm{L}$ of methanol-water $(1: 1, v / v)$ and ochratoxin A content was determined by HPLC-FLD. Under optimized extraction conditions, the limit of detection of $0.9 \mu \mathrm{g} \cdot \mathrm{kg}^{-1}$ and $2.5 \mu \mathrm{g} \cdot \mathrm{kg}^{-1}$ and the precision of $3.4 \%$ and $5.0 \%$ over a concentration range of 1 to $100 \mu \mathrm{g} \cdot \mathrm{kg}^{-1}$ in wheat and maize flour, respectively, were obtained.
\end{abstract}


Keywords: ochratoxin A; solid phase microextraction (SPME); solid bar microextraction (SBME)

\section{Introduction}

Mycotoxin ochratoxin A (OTA) is produced by numerous Penicillium and Aspergillus species such as Penicillium verrucosum, Penicillium nordicum, Aspegillus ochraceus, and Aspergillus carbonariusm, while new producers are continuously being discovered [1]. OTA occurs ubiquitously in plant products such as cereals, beans, groundnuts, raisins, coffee, beer and wine, as well as in certain animal products [2]. Cereals (wheat, barley, and oats) are the main source of human exposure to OTA [3]. Feedstuff is frequently contaminated with OTA, too; the levels vary with country and commodity, cereals being the most frequently-contaminated feed ingredient [4]. Interaction among OTA producers and other fungi suppresses or stimulates OTA production [5]. OTA has allegedly been implicated in a range of toxicological effects, including nephrotoxicity, mutagenicity, teratogenicity, neurotoxicity, and immunotoxicity, in animals and humans [6]. The mode of action appears to be associated with oxidative damage [7], though the effect OTA on epigenetic control has recently been demonstrated [8]. The widespread occurrence of OTA in food motivated continuous improvement of analytical methods for OTA during the last decade.

Liquid chromatography with fluorescence detection (LC-FLD) is the most used chromatographic technique for OTA determination [9-13]. Immunochemical methods [14] and thin-layer chromatography [15] offer high throughput due to the parallel analysis of many samples, while HPLC, with mass spectrometric detection, allows analyzing many mycotoxins simultaneously [16,17]. In spite of the advantages of alternative methods, LC-FLD remains the most popular method for OTA determination due to its high sensitivity and relatively inexpensive equipment available in most analytical laboratories. Sample pretreatment consisting of extraction, clean-up, and often preconcentration is required to remove matrix components and enhance sensitivity $[18,19]$. Common extraction methods are based on organic solvents such as acetonitrile, methanol, chloroform, and ethyl acetate, which are often acidified. The standard technique for clean-up and preconcentration of OTA is solid-phase extraction (SPE) [19]. A variety of SPE columns have been used including home-made columns filled with C8 sorbent [8], C18 column followed by cleanup on a mixed-mode polymer sorbent column [20], mixed-mode dispersive SPE [18], silica [11], molecularly-imprinted polymers [21], and immunoaffinity columns [9,13,22]. Liquid-liquid extraction (LLE) is also commonly used, often combined with SPE [9,11]. Interesting new developments include the use of ionic liquids as extraction solvents in LLE [18], cleanup of OTA by coacervation of reverse micelles [23], and liquid-liquid microextraction in porous hollow fibers [24]. Both SPE (except for the immunoaffinity columns) and LLE in a traditional setup require multistep protocols that are time-consuming and use large volumes of organic solvents; see [25] for a summary of the drawbacks. Immunoaffinity columns (IAC) offer unsurpassed specificity but are expensive, nor recyclable, have a limited binding capacity (heavily contaminated samples have to be diluted and submitted to a second cleanup, spending another IAC column), and a limited shelf life. The development of new simple, inexpensive, and environmentally 
friendly extraction and purification protocols therefore remains an important task in the analysis of OTA content in foodstuff.

Solvent-minimized solid phase microextraction (SPME) technique has been used for the determination of OTA in beer [26], coffee beans [27], cornflakes [28], and cheese [29]. SPME is a sorbent-based method in which extracting media is limited to $\mu \mathrm{m}$-scale-sized sorbent, physically or chemically coated on fused-silica fiber [30]. A drawback of the technique is that SPME fibers are expensive and their lifetime is limited [25].

The recently developed solid bar microextraction (SBME) as an alternative miniaturized micro-solid phase extraction technique [31] that overcomes these difficulties by using only a few milligrams of a sorbent wrapped in a hollow fiber micro-tube [26,32,33]. Due to the porosity of the membrane, analytes are able to diffuse through and adsorb to the sorbent. Tumbling the solid "bar" in sample solution by stirring facilitates the extraction process. Porous membrane acts as a filter, excluding particles from the sample matrix from access to the sorbent. After the extraction, analytes are desorbed by immersing the device in a suitable organic solvent.

The most recent advancement in the determination of OTA was the use of aptamers. In spite of the promising results [34-36], the technique is not mature yet to be introduced into routine analytical practice. Immunochemical techniques for the determination of multiple mycotoxins in a single analysis were recently enhanced by the introduction of planar waveguides but the sensitivity remains a problem [37].

In this work, we investigated the application of SBME combined with HPLC-FLD to determine OTA content in wheat and maize grains. Parameters affecting the extraction efficiency such as sorbent properties, $\mathrm{pH}$ of the extract, extraction time, and composition of the desorption solvents were studied. Since cereal grains are the main source of OTA exposure [3], and maize is a staple grain in many countries, we have chosen wheat and maize as matrices for which the performance parameters of the method were determined.

\section{Results and Discussion}

\subsection{Optimization of SBME Conditions}

Acetonitrile-water extraction is the standard sample preparation for OTA determination in wheat and maize [13,16]. Elimination of acetonitrile before SBME is necessary; initial studies were therefore performed with the analyte dissolved in ultrapure water. The following SPME parameters were optimized for maximum OTA recovery: type of sorbent, number of SBME devices used, sample $\mathrm{pH}$, extraction time, stirring speed, and desorption conditions. Concentrations of OTA of 4 to $5 \mu \mathrm{g} \mathrm{L}{ }^{-1}$ were used in most optimization experiments in line with the maximum level allowed in raw cereals. Lower concentrations of 1 to $2 \mu \mathrm{g} \mathrm{L}{ }^{-1}$ were used in experiments involving time course analysis to assure that the optimized time will be sufficient for lowest concentrations analyzed because the rate of adsorption/desorption is proportional to concentration. Optimization experiments were performed in triplicate. In line with IUPAC recommendation, extraction efficiency (recovery factor) was determined as the yield of OTA after extraction and apparent recovery was determined as the ratio of the peak area of a spiked sample, corrected for the background, to the peak area of the signal of pure standard [38]. 
The selection of an appropriate sorbent material is of major importance for the optimization of the SBME process. C4, C8, C18 and endcapped C18ec were compared in the extraction of OTA from $5 \mathrm{~mL}$ water samples. After extraction, the SBME devices were desorbed in methanol and the residue reconstituted in $50 \mu \mathrm{L}$ of mobile phase and analyzed. As shown in Figure 1, C18 and C18ec showed the highest extraction efficiency; non-endcapped C18 was chosen as sorbent for further experiments.

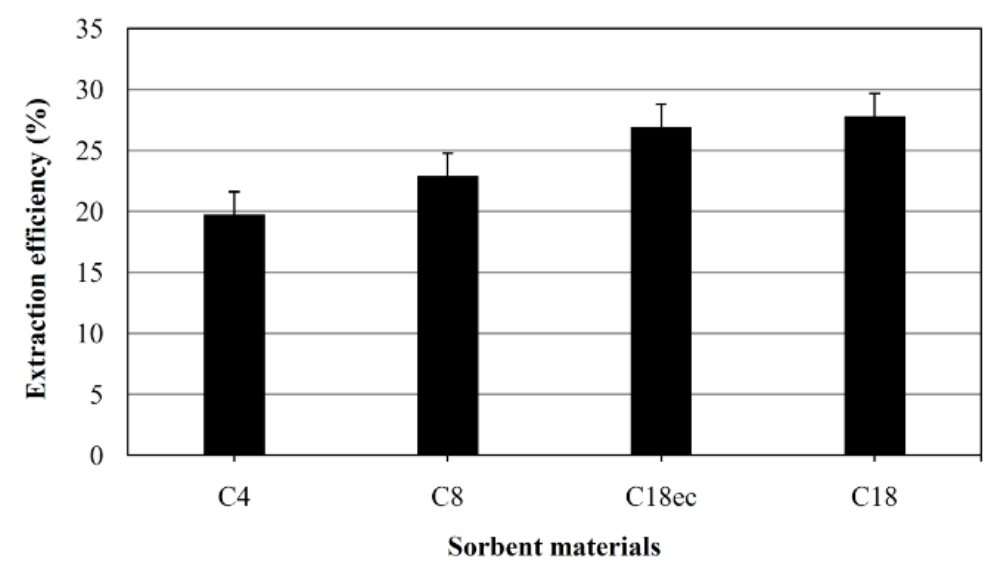

Figure 1. Effect of sorbent materials on SBME efficiency. Extraction conditions: $5 \mu \mathrm{g} \mathrm{L} \mathrm{L}^{-1}$ of OTA in $5 \mathrm{~mL}$ water, extraction time $90 \mathrm{~min}$, stirring speed $500 \mathrm{rpm}$, desorption into $150 \mu \mathrm{L}$ methanol in $10 \mathrm{~min}$; three devices were used in each extraction. Error bars correspond to standard deviation.

The effect of using multiple SBME devices was evaluated by comparing extraction efficiency achieved with 1 to 7 devices for OTA solutions of $3 \mu \mathrm{g} \mathrm{L}^{-1}$ dissolved in $0.01 \mathrm{M} \mathrm{HCl}$. As expected, extraction efficiency increased with the number of devices (Figure 2). However, when more than five devices were used, no additional enhancement was observed. Thus, five SBME devices were used for the remaining studies. Because OTA is a weak acid, samples for the extraction of OTA into organic solvents or for binding of OTA on hydrophobic matrices are usually acidified. $\mathrm{pH}$ of 2.0 was selected in our work.

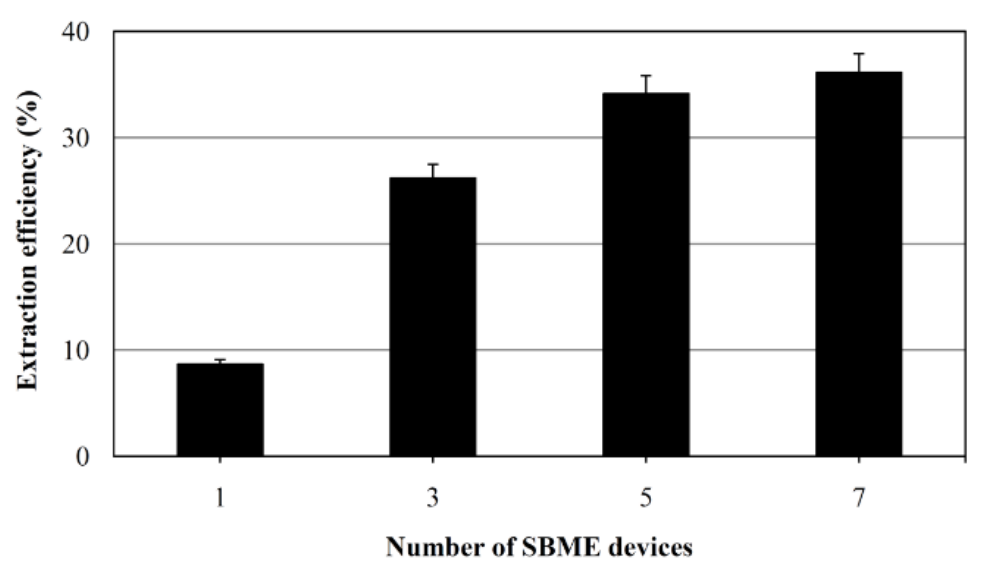

Figure 2. Effect of the number of SBME devices on extraction efficiency. Extraction conditions: $5 \mu \mathrm{g} \mathrm{L}^{-1}$ of OTA in $5 \mathrm{~mL}$ of $10 \mathrm{mM} \mathrm{HCl}, \mathrm{C} 18$ sorbent, extraction time $70 \mathrm{~min}$, stirring speed $300 \mathrm{rpm}$, desorption into $150 \mu \mathrm{L}$ methanol in $15 \mathrm{~min}$; three devices were used in each extraction. Error bars correspond to standard deviation. 
Adsorption of OTA on SBME sorbent is an equilibrium process. In order to determine the extraction time needed to achieve equilibrium, we extracted a solution of $1 \mu \mathrm{g} \mathrm{L}^{-1}$ of OTA in $0.01 \mathrm{M} \mathrm{HCl}$ for 10 to 90 min. As illustrated in Figure 3, the extraction reached equilibrium after 70 min. Thus, 70 min was selected for as the extraction time.

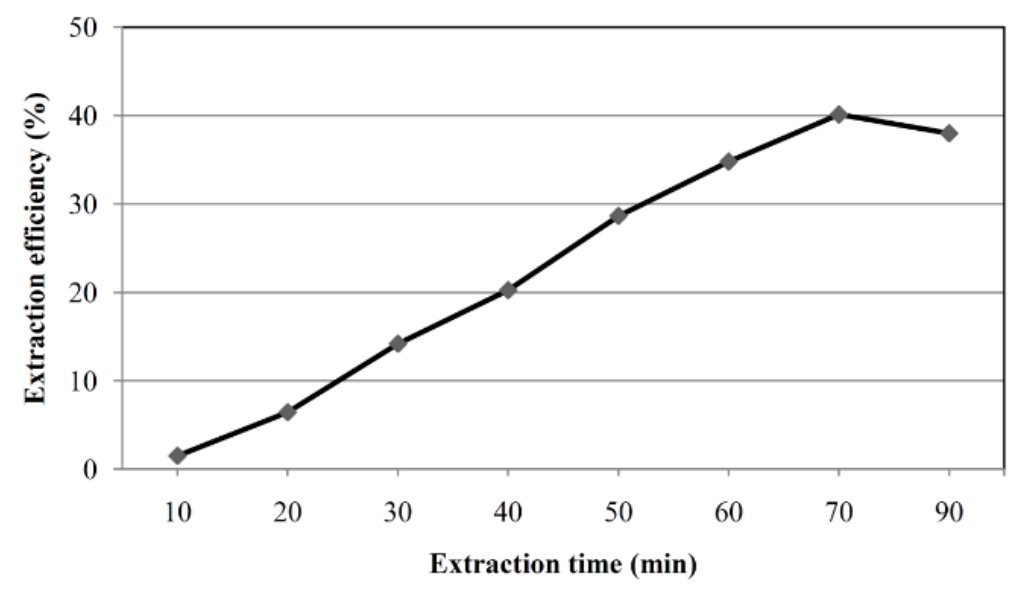

Figure 3. Kinetics of the OTA adsorption on SPME device. Conditions as in Figure 2, except for the concentration of OTA which was $1 \mu \mathrm{g} \mathrm{L}{ }^{-1}$.

Stirring reduces the time at which adsorption equilibrium is reached. Comparison of stirring rates of 100, 500, and $1000 \mathrm{rpm}$ (Figure 4) showed that the highest adsorption was achieved at $500 \mathrm{rpm}$. Stirring at $1000 \mathrm{rpm}$ caused formation of bubbles which tended to adhere to the surface of the fiber, impeding OTA transfer. Stirring at $500 \mathrm{rpm}$ was chosen for further studies.

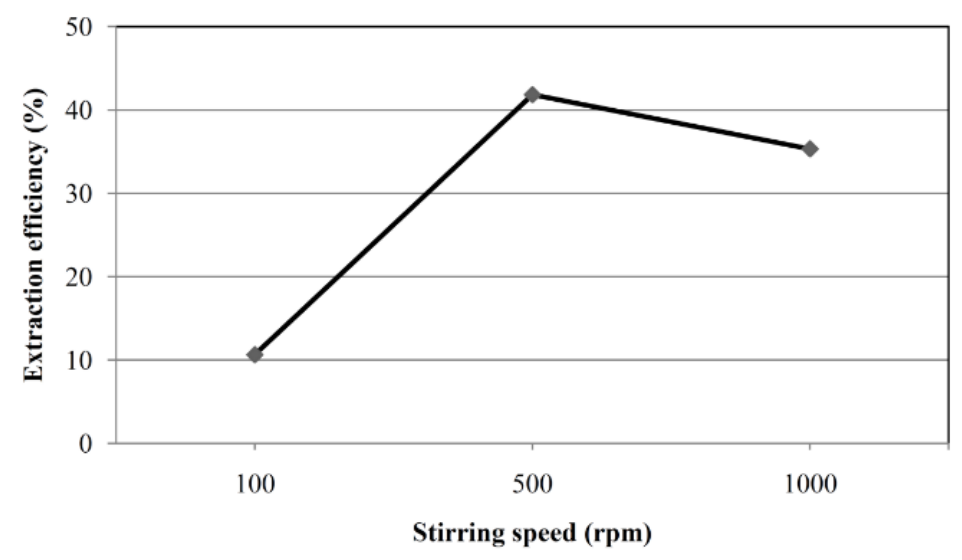

Figure 4. Influence of stirring speed on the recovery. OTA at a concentration of $4 \mu \mathrm{g} \mathrm{L}-1$ was used, for other conditions refer to Figure 2.

Selection of a suitable desorption conditions were also evaluated. Methanol, acetonitrile and acetone were used with sonication to release OTA from the sorbent. Methanol showed the best results (results not shown). Sonication time was also varied from 5 to $20 \mathrm{~min}$. Figure 5 depicts the desorption profile of OTA (spiked at $2 \mu \mathrm{g} \mathrm{L}^{-1}$ ) showing that $15 \mathrm{~min}$ desorption time gave satisfactory results. No carryover of the analyte was observed when the SBME device after desorption was used again, which means that the devices are reusable. SBME devices were reused up to 20 times without compromising extraction efficiency (results not shown). 


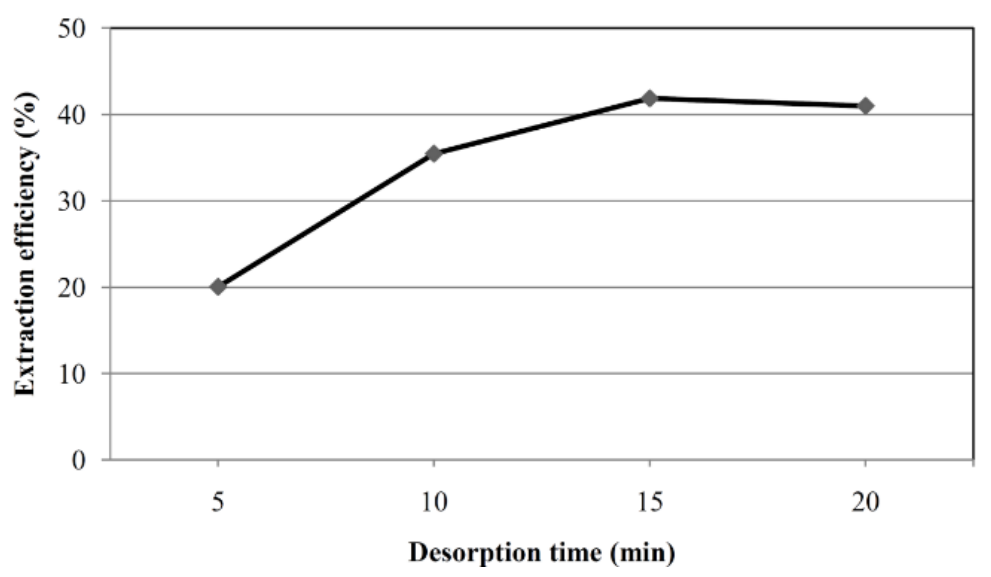

Figure 5. Effect of desorption time on the recovery of OTA in SBME. OTA at a concentration of $2 \mu \mathrm{g} \mathrm{L}{ }^{-1}$ was used, for other conditions refer to Figure 2.

\subsection{Analysis of Maize and Wheat Samples}

Because wheat and maize grains are rich in fat which may compete with OTA for binding on SBME and compromise the performance of HPLC, acetonitrile-water extracts of ground grains were defatted with hexane (see Material and Method) prior to SPME cleanup.

To quantify losses of OTA during sample pre-treatment, a procedure similar to one described by [27] was used. Two $5 \mathrm{~g}$ aliquots of ground, OTA-free wheat and maize grains were processed. The first aliquot was spiked with $2.5 \mu \mathrm{L}$ of a $1 \mathrm{mg} \mathrm{mL}^{-1}$ OTA standard solution and left to equilibrate as described earlier (see "Sample Preparation"). Complete recovery would imply a final OTA concentration of $167 \mu \mathrm{g} \mathrm{L}^{-1}$ in $15 \mathrm{~mL}$ of acetonitrile-water-acetic acid extract. The second aliquot was processed as the first one, except that OTA was spiked into the final extract. Experiments were performed in triplicate. Peak areas of OTA signal of spiked sample, as compared to spiked extracts (apparent recoveries), were $97 \% \pm 6 \%$ for wheat and $98 \% \pm 4 \%$ for maize. The chromatographic conditions allowed a satisfactory separation of OTA from matrix components detectable with FLD at the same excitation/emission wavelengths in less than $6 \mathrm{~min}$, as shown in a representative chromatogram in Figure 6.

The overall recoveries of OTA at a concentration of $5 \mu \mathrm{g} \cdot \mathrm{kg}^{-1}$ were $35 \%$ and $38 \%$ for wheat and maize, respectively. At the spiking level of $50 \mu \mathrm{g} \cdot \mathrm{kg}^{-1}$, recoveries of $36 \%$ and $39 \%$ for wheat and maize, respectively, were obtained. These values would be unacceptably low for standard SPE or LLE but they are common in non-exhaustive microextraction methods. For example, the recovery of OTA from beer by SPME under recommended conditions (60 min under stirring) was only $15 \%$ and the adsorption time would have to be extended to $10 \mathrm{~h}$ to reach equilibrium with a recovery of $60 \%$ [26]. Liquid-liquid-microextraction, too, suffers from long adsorption times and low recoveries [24]. The laboratory where SPME was invented has recently used the technique for the determination of OTA in cheese [29]; because their protocol is based on $50 \mathrm{~mL}$ loading solution, they had to increase the adsorption time to $8 \mathrm{~h}$. In our protocol the time to equilibrium is much shorted (cf. Figure 3) because loading volume is 10-times smaller, but the low recovery is a problem that has to be addressed by future research. Even cleanup of OTA extracts based on standard-size SPE columns commonly lead to recoveries as low as $70 \%$, as shown in a recent method comparison [10]. 
Performance parameters of the methods are listed in Table 1. LODs and LOQs were calculated with the Valoo software (Applica, Bremen, Germany) based on the standardization criteria DIN 32645 as defined by the German standardization committee [39]. LODs in the range of 0.92 and $2.48 \mu \mathrm{g} \cdot \mathrm{kg}^{-1} \mathrm{are}$ below the tolerance level for OTA in raw cereals permitted by EU directives $\left(5.0 \mu \mathrm{g} \cdot \mathrm{kg}^{-1}\right)$ but the LOQ for maize is above this limit. The high sensitivity required for OTA analysis due to low regulatory limits poses a challenge to current analytical technology. A recently-published method for OTA in wine, which is an easier matrix than wheat and maize, generated comparable chromatograms for samples with the same level of OTA [18] (compare Figure 6 with Figure 4 in the cited publication), though a more sensitive laser-induced fluorescence detector was used.

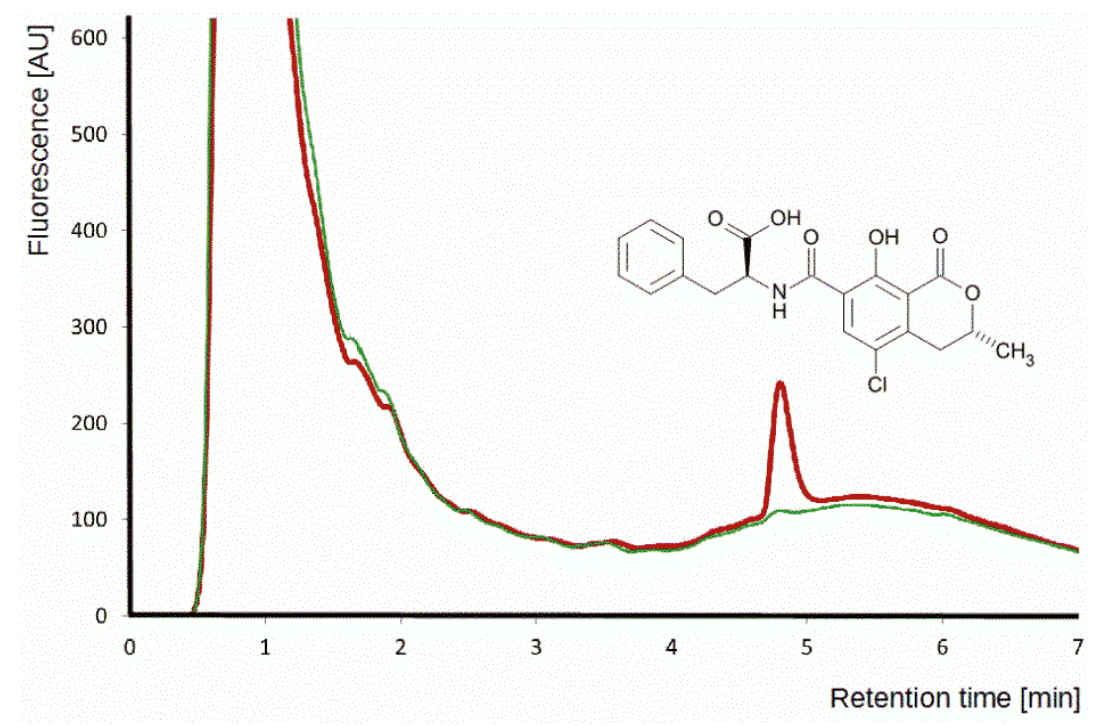

Figure 6. HPLC-FLD chromatogram obtained after SBME of wheat spiked with OTA at $10 \mathrm{ng} \mathrm{g}^{-1}$ (red) and wheat extract not containing detectable amounts of OTA (green). Fluorescence intensity in arbitrary units was plotted against retention time. The structure of OTA is shown above the peak of the mycotoxin at $4.9 \mathrm{~min}$.

Table 1. Performace parameters of SBME for OTA in wheat and maize grains.

\begin{tabular}{ccc}
\hline Performance parameter & Wheat & Maize \\
\hline LOD & $2.5 \mu \mathrm{g} \cdot \mathrm{kg}^{-1}$ & $0.9 \mu \mathrm{g} \cdot \mathrm{kg}^{-1}$ \\
LOQ & $8.7 \mu \mathrm{g} \cdot \mathrm{kg}^{-1}$ & $3.4 \mu \mathrm{g} \cdot \mathrm{kg}^{-1}$ \\
Correlation coefficient $(r)^{*}$ & 0.995 & 0.998 \\
Repeatability as RSD $(n=5)$ & $5.0 \%$ & $3.4 \%$ \\
\hline
\end{tabular}

* The coefficient of correlation was calculated for OTA at concentrations $5,10,25,50$ and $100 \mu \mathrm{g} \cdot \mathrm{kg}^{-1}$.

Calibration curves for direct injection of OTA extracts without SBME enrichment were also constructed (data not shown); the LOD values found were about 100 times higher than the values reported in Table 1, indicating a large increase of sensitivity provided by SBME. Repeatability of the method was comparable or better than published methods based on liquid-liquid microextraction followed by HPLC-FD [18] and SPME followed by HPLC-MS/MS [29] but not as good as in a method that included double cleanup by chloroform partition and SPME followed by HPLC-FD [27]. 


\section{Experimental Section}

\subsection{Chemicals and Reagents}

Analytical standard of ochratoxin $\mathrm{A}\left(1 \mathrm{mg} \mathrm{mL} \mathrm{m}^{-1}\right.$ in acetonitrile) was purchase from Fermentek (Jerusalem, Israel). Sorbent materials Chromabond C4 (C4), Chromabond C8 (C8), Chromabond C18 (C18), and Chromabond $\mathrm{C} 18$ endcapped (C18ec) were obtained from Macherey-Nagel (Düren, Germany). Hydrochloric acid (37\%) and HPLC-grade organic solvents were obtained from Merck (Darmstadt, Germany). Q3/2 Accurel polypropylene hollow fiber membrane (600 $\mu \mathrm{m}$ i.d., $200 \mu \mathrm{m}$ wall thickness, and $0.2 \mu \mathrm{m}$ pore size) was purchased from Membrana (Wuppertal, Germany).

\subsection{Sample Preparation}

Wheat and maize grains were purchased in a local supermarket. Ground kernels ( $5 \mathrm{~g})$ were spiked by adding the appropriate amounts of OTA in methanol to the flour. The samples were subsequently stored for three days at $40{ }^{\circ} \mathrm{C}$ to allow evaporation of the methanol and establish equilibrium between OTA and the matrix, simulating natural contamination. The samples were extracted with $15 \mathrm{~mL}$ of acetonitrile-water-acetic acid $(79: 20: 1, v / v / v)$ for 12 hours on a shaker (100 rpm) at laboratory temperature and subsequently centrifuged at $1500 \mathrm{rpm}$ for $10 \mathrm{~min}$. The supernatants were defatted with $5 \mathrm{~mL}$ cyclohexane. Extraction solvent was dried in vacuum for $4 \mathrm{~h}$ at $45^{\circ} \mathrm{C}$. Dry residue was dissolved in $5 \mathrm{~mL} 0.01 \mathrm{M} \mathrm{HCl}$ and the solution was filtrated through 55-mm diameter GF/A glass microfiber filter (Whatman, Maidstone, UK). The solution was transferred into a glass vial of $10 \mathrm{~mL}$ and subjected to SBME.

\subsection{Solid Bar Microextraction Procedure}

The SBME device consists of sorbent materials enclosed within a hollow fiber polypropylene micro-tube (HF-PPMT). The SBME device was prepared as follows: HF-PPMT was manually cut with a sharp knife (scalpel blade) into pieces of $2.5 \mathrm{~cm}$ length. Each piece was closed from one side by means of a hot soldering tool, washed with methanol in an ultrasonic cleaner, and dried. A rod of stainless steel ( $0.4 \mathrm{~mm}$ diameter and $10 \mathrm{~cm}$ length) was used to introduce sorbent ( $\sim 2 \mathrm{mg})$ through the open end into the lumen of the fiber. After filling with sorbent, the remaining open end of the tube was then heat-sealed to secure the contents. The SBME device was cleaned by sonication in methanol for 3 min and then stored in methanol until use.

A clean SBME device was placed in a $10 \mathrm{~mL}$ vial with sample extract (see sample preparation), and stirred at different speeds. The device tumbled freely in the sample during the extraction. After the extraction, the device was removed with a pair of tweezers, dried with lint-free tissue and placed in a $250 \mu \mathrm{L}$ HPLC micro-vial containing $200 \mu \mathrm{L}$ methanol. Desorption of OTA was facilitated by sonication. After removing the SPME device, the sample was dried in vacuum. The residue was reconstituted in $50 \mu \mathrm{L}$ of methanol-water $(1: 1, v / v)$ and $20 \mu \mathrm{L}$ were injected into HPLC-FLD unit for analysis. 


\subsection{Chromatography}

The HPLC system consisted of a JASCO PU-2080 plus ternary pump, JASCO AS-2059-SF autosampler and a JASCO FP-2020 florescence detector (Jasco, Gotha, Germany). JASCO ChromPass chromatography data system (version 1.8.6.1; Jasco, Gotha, Germany) was used for data processing. Chromatographic separation was performed at $25{ }^{\circ} \mathrm{C}$ on a Kinetex ${ }^{\mathrm{TM}} \mathrm{C} 18$ column, $50 \times 4.6 \mathrm{~mm}$, $2.6 \mu \mathrm{m}$ particle size, equipped with a C18, $4 \times 3 \mathrm{~mm}$ pre-column (Phenomenex, Torrance, CA, USA). Eluents containing $7 \mathrm{mM}$ acetic acid were acetonitrile-water (95:5, $v / v$, eluent A) or methanol (eluent B). The flow rate was $1 \mathrm{~mL} \mathrm{~min}{ }^{-1}$. After an initial $0.2 \mathrm{~min}$ at $45 \% \mathrm{~A}$, the proportion of B increased linearly to $98 \%$ within $10 \mathrm{~min}$, followed by a washing step for $2 \mathrm{~min}$ at $98 \% \mathrm{~B}$ and 5 min equilibration at $45 \%$ A. The fluorescence detector was set up at excitation and emission wavelength of 333 and $455 \mathrm{~nm}$, respectively. Injection volume of $20 \mu \mathrm{L}$ was used throughout the study. Quantification was performed by integrating peak areas. Calibration curves for OTA were constructed in the range of $0.05-1.0 \mu \mathrm{g} \mathrm{L}{ }^{-1}$.

\section{Conclusions}

SBME followed by LC-FLD was developed for the determination of OTA in wheat and maize samples. The method exhibits good precision and linear response over a wide concentration range. The apparent recovery was low $(35 \%-39 \%)$ due to the non-exhaustive extraction technique used. Under optimal extraction conditions, LODs of $1.84 \mathrm{ng}$ OTA on column for maize and $5.0 \mathrm{ng}$ OTA on column for wheat were obtained, corresponding to $0.92 \mu \mathrm{g} \cdot \mathrm{kg}^{-1}$ and $2.5 \mu \mathrm{g} \cdot \mathrm{kg}^{-1}$ in maize and wheat, respectively. The method consumes a low amount of organic solvents, is easy to use, and cheaper than most currently-used cleanup procedures for OTA. Due to its low apparent recovery and high LOQ for wheat, the method is not suitable for official control of OTA level according to European legislative.

\section{Acknowledgments}

Nabil AL-Hadithi thanks the Georg-August-University Göttingen for providing a scientist scholar research position. The study was supported by FAEN Joint Project "Quality-related plant production under modified basic conditions: mycotoxins in the context of production, quality and processing" funded by the Ministry of Science and Culture of Lower Saxony, Germany.

\section{Author Contributions}

Nabil AL-Hadithi carried out SBME experiments; Philip Kössler carried out OTA analysis by LC-FD; Petr Karlovsky supervised the project and guided the characterization of the method; Nabil AL-Hadithi wrote the first version of the manuscript; Philip Kössler contributed the description of LC-FD; Petr Karlovsky revised the manuscript before submission.

\section{Conflicts of Interest}

The authors declare no conflict of interest. 


\section{References}

1. Davolos, D.; Pietrangeli, B.A. Molecular and bioinformatic study on the ochratoxin A (OTA)-producing Aspergillus affinis (section Circumdati). Mycotoxin Res. 2014, 30, 113-122.

2. Jørgensen, K. Occurrence of ochratoxin A in commodities and processed food-A review of EU occurrence data. Food Addit. Contam. 2005, 22 (Suppl. 1), 26-30.

3. Duarte, S.C.; Pena, A.; Lino, C.M. A review on ochratoxin A occurrence and effects of processing of cereal and cereal derived food products. Food Microbiol. 2010, 27, 187-198.

4. Streit, E.; Schatzmayr, G.; Tassis, P.; Tzika, E.; Marin, D.; Taranu, I.; Tabuc, C.; Nicolau, A.; Aprodu, I.; Puel, O.; et al. Current situation of mycotoxin contamination and co-occurrence in animal feed-Focus on Europe. Toxins 2012, 4, 788-809.

5. Barberis, C.L.; Pena, G.; Carranza, C.; Magnoli, C.E. Effect of indigenous mycobiota on ochratoxin A production by Aspergillus carbonarius isolated from soil: Ochratoxin in mixed cultures. Mycotoxin Res. 2014, 30, 1-8.

6. O’Brien, E.; Dietrich, D.R. Ochratoxin A: The continuing enigma. Crit. Rev. Toxicol. 2005, 35, 33-60.

7. Sorrenti, V.; Di Giacomo, C.; Acquaviva, R.; Barbagallo, I.; Bognanno, M.; Galvano, F. Toxicity of ochratoxin A and its modulation by antioxidants: A review. Toxins 2013, 5, 1742-1766.

8. Oh, S.-Y.; Balch, C.G.; Cliff, R.L.; Sharma, B.S.; Boermans, H.J.; Swamy, H.V.L.N.; Quinton, V.M.; Karrow, N.A. Exposure to Penicillium mycotoxins alters gene expression of enzymes involved in the epigenetic regulation of bovine macrophages (BoMacs). Mycotoxin Res. 2013, 29, 235-243.

9. Blesa, J.; Berrada, H.; Soriano, J.M.; Moltó, J.C.; Mañes, J. Rapid determination of ochratoxin A in cereals and cereal products by liquid chromatography. J. Chromatogr. A 2004, 1046, 127-131.

10. Dall'asta, C.; Galaverna, G.; De Dea Lindner, J.; Virgili, R.; Neviani, E.; Dossena, A.A. New validated HPLC-FLD method for detecting ochratoxin A in dry-cured meat and in blue cheese. Mycotoxin Res. 2007, 23, 132-137.

11. Lindenmeier, M.; Schieberle, P.; Rychlik, M. Determination of ochratoxin A in food: comparison of a stable isotope dilution assay, liquid chromatography-fluorescence detection and an enzyme-linked immunosorbent assay. Mycotoxin Res. 2011, 27, 115-121.

12. Medina, A.; Valle-Algarra, F.M.; Gimeno-Adelantado, J.V.; Mateo, R.; Mateo, F.; Jiménez, M. New method for determination of ochratoxin A in beer using zinc acetate and solid-phase extraction silica cartridges. J. Chromatogr. A 2006, 1121, 178-183.

13. Reiter, E.V.; Cichna-Markl, M.; Chung, D.H.; Shim, W.B.; Zentek, J.; Razzazi-Fazeli, E. Determination of ochratoxin A in grains by immuno-ultrafiltration and HPLC-fluorescence detection after postcolumn derivatisation in an electrochemical cell. Anal. Bioanal. Chem. 2011, 400, 2615-2622.

14. Meulenberg, E.P. Immunochemical methods for ochratoxin A detection: A review. Toxins 2012, 4, 244-266.

15. Ventura, M.; Anaya, I.; Broto-Puig, F.; Agut, M.; Comellas, L. Two-dimensional thin-layer chromatographic method for the analysis of ochratoxin A in green coffee. J. Food Prot. 2005, 68, 1920-1922.

16. Schenzel, J.; Forrer, H.R.; Vogelgsang, S.; Bucheli, T.D. Development, validation and application of a multi-mycotoxin method for the analysis of whole wheat plants. Mycotoxin Res. 2012, 28, 135-147. 
17. Sulyok, M.; Berthiller, F.; Krska, R.; Schuhmacher, R. Development and validation of a liquid chromatography/tandem mass spectrometric method for the determination of 39 mycotoxins in wheat and maize. Rapid Commun. Mass Spectrom. 2006, 20, 2649-2659.

18. Arroyo-Manzanares, N.; García-Campaña, A.M.; Gámiz-Gracia, L. Comparison of different sample treatments for the analysis of ochratoxin A in wine by capillary HPLC with laser-induced fluorescence detection. Anal. Bioanal. Chem. 2011, 401, 2987-2994.

19. Monaci, L.; Palmisano, F. Determination of ochratoxin A in foods: State-of-the-art and analytical challenges. Anal. Bioanal. Chem. 2004, 378, 96-103.

20. Chiodini, A.M.; Scherpenisse, P.; Bergwerff, A.A. Ochratoxin a contents in wine: comparison of organically and conventionally produced products. J. Agric. Food Chem. 2006, 54, 7399-7404.

21. Zhou, S.N.; Lai, E.P.C.; Miller, J.D. Analysis of wheat extracts for ochratoxin A by molecularly imprinted solid-phase extraction and pulsed elution. Anal. Bioanal. Chem. 2004, 378, 1903-1906.

22. Turcotte, A.-M.; Scott, P.M.; Tague, B. Analysis of cocoa products for ochratoxin A and aflatoxins. Mycotoxin Res. 2013, 29, 193-201.

23. García-Fonseca, S.; Ballesteros-Gómez, A.; Rubio, S.; Pérez-Bendito, D. Coacervative extraction of Ochratoxin A in wines prior to liquid chromatography/fluorescence determination. Anal. Chim. Acta 2008, 617, 3-10.

24. González-Peñas, E.; Leache, C.; Viscarret, M.; Pérez de Obanos, A.; Araguás, C.; López de Cerain, A. Determination of ochratoxin $\mathrm{A}$ in wine using liquid-phase microextraction combined with liquid chromatography with fluorescence detection. J. Chromatogr. A. 2004, 1025, 163-168.

25. Jiang, X.; Lee, H.K. Solvent bar microextraction. Anal. Chem. 2004, 76, 5591-5596.

26. Aresta, A.; Palmisano, F.; Vatinno, R.; Zambonin, C.G. Ochratoxin A determination in beer by solid-phase microextraction coupled to liquid chromatography with fluorescence detection: A fast and sensitive method for assessment of noncompliance to legal limits. J. Agric. Food Chem. 2006, 54, 1594-1598.

27. Vatinno, R.; Aresta, A.; Zambonin, C.G.; Palmisano, F. Determination of ochratoxin A in green coffee beans by solid-phase microextraction and liquid chromatography with fluorescence detection. J. Chromatogr. A. 2008, 1187, 145-150.

28. Aresta, A.; Cioffi, N.; Palmisano, F.; Zambonin, C.G. Simultaneous determination of ochratoxin A and cyclopiazonic, mycophenolic, and tenuazonic acids in cornflakes by solid-phase microextraction coupled to high-performance liquid chromatography. J. Agric. Food Chem. 2003, 51, 5232-5237.

29. Zhang, X.; Cudjoe, E.; Vuckovic, D.; Pawliszyn, J. Direct monitoring of ochratoxin A in cheese with solid-phase microextraction coupled to liquid chromatography-tandem mass spectrometry. J. Chromatogr. A. 2009, 1216, 7505-7509.

30. Zambonin, C.G. Coupling solid-phase microextraction to liquid chromatography. A review. Anal. Bioanal. Chem. 2003, 375, 73-80.

31. Basheer, C.; Chong, H.G.; Hii, T.M.; Lee, H.K. Application of porous membrane-protected micro-solid-phase extraction combined with HPLC for the analysis of acidic drugs in wastewater. Anal. Chem. 2007, 79, 6845-6850.

32. AL-Hadithi, N.; Saad, B.; Grote, M. A solid bar microextraction method for the liquid chromatographic determination of trace diclofenac, ibuprofen and carbamazepine in river water. Microchim. Acta 2011, 172, 31-37. 
33. Lee, T.P.; Saad, B.; Khayoon, W.S.; Salleh, B. Molecularly imprinted polymer as sorbent in micro-solid phase extraction of ochratoxin A in coffee, grape juice and urine. Talanta 2012, 88 , 129-135.

34. Rhouati, A.; Yang, C.; Hayat, A.; Marty, J.-L. Aptamers: A promising tool for ochratoxin A detection in food analysis. Toxins 2013, 5, 1988-2008.

35. Wei, Y.; Zhang, J.; Wang, X.; Duan, Y. Amplified fluorescent aptasensor through catalytic recycling for highly sensitive detection of ochratoxin A. Biosens. Bioelectron. 2014, 65, 16-22.

36. Sanzani, S.M.; Reverberi, M.; Fanelli, C.; Ippolito, A. Detection of ochratoxin a using molecular beacons and real-time PCR thermal cycler. Toxins 2015, 7, 812-820.

37. Tittlemier, S.A.; Roscoe, M.; Drul, D.; Blagden, R.; Kobialka, C.; Chan, J.; Gaba, D. Single laboratory evaluation of a planar waveguide-based system for a simple simultaneous analysis of four mycotoxins in wheat. Mycotoxin Res. 2013, 29, 55-62.

38. Burns, D.T.; Danzer, K.; Townshend, A. Use of the term "recovery" and "apparent recovery" in analytical procedures. (IUPAC Recommendations 2002). Pure Appl. Chem. 2002, 74, 2201-2205.

39. Kaus, R. Detection limits and quantitation limits in the view of international harmonization and the consequences for analytical laboratories. Accred. Qual. Assur. 1998, 3, 150-154.

(C) 2015 by the authors; licensee MDPI, Basel, Switzerland. This article is an open access article distributed under the terms and conditions of the Creative Commons Attribution license (http://creativecommons.org/licenses/by/4.0/). 